\title{
Über substituierte Rhodaninsäuren und deren Aldehydkondensationsprodukte
}

\author{
(II. Mitteilung) \\ von
}

Rudolf Andreasch und Dr. Arthur Zipser.

(Vorgelegt in der Sitzung am 3. Dezember 1903.)

Wir haben vor einiger Zeit ${ }^{1}$ mitgeteilt, daß sich Senföle mit Thioglykolsäure zu am Stickstoff substituierten Rhodaninsäuren verbinden; in dieser Art wurde v-Phenyl- und v-Allylrhodaninsäure dargestellt.

Ein Körper von der Zusammensetzung der v-Phenylrhodaninsäure wurde auch bereits von v. Braun ${ }^{2}$ aus phenyldithiokarbaminsaurem Ammonium durch Einwirkung von Bromessigsäureäthylester erhalten. Wir haben uns diese Verbindung ebenfalls nach der Methode von v. Braun dargestellt, nur mit dem Unterschiede, daß wir den Bromessigester durch Chloressigester ersetzten. Beide Verbindungen erwiesen sich in allen Punkten identisch.

Zur Darstellung der Phenylrhodaninsäure wurde das fein geriebene phenyldithiokarbaminsaure Ammonium in Alkohol suspendiert und mit der berechneten Menge von Chloressigsäureäthylester versetzt; đabei tritt Erwärmung ein. Durch Erhitzen am Wasserbade wird die Reaktion zu Ende geführt, das Reaktionsgemisch dann mit Wasser versetzt zur Lösung des gebildeten Salmiaks und der abgeschiedene Krystallkuchen nach dem Trocknen aus heißem Alkohol oder siedendem Aceton umkrystallisiert.

\footnotetext{
1 Monatshefte für Chemie, 24, 499 bis 518.

2 Berichte der Deutschen chem. Gesellsch., 35, 3387.
} 
Es braucht wohl nicht hervorgehoben werden, daß diese v. Braun'sche Methode zur Beschaffung größerer Mengen von Phenylrhodaninsäure wegen ihrer raschen Ausführbarkeit und Billigkeit den Vorzug vor der von uns angegebenen verdient.

Von den 1. c. beschriebenen Rhodaninsäuren wurden noch folgende Aldehydkondensationsprodukte dargestellt, wobei bemerkt werden soll, daß zur Kondensation in eisessigsaurer Lösung der Zusatz von essigsaurem Natron überflüssig erscheint; wir haben bei Weglassung dieser Substanz keine schlechteren Ausbeuten erzielt als mit derselben.

Es wurde also stets der Aldehyd und die betreffende Rhodaninsäure in äquimolekularem Verhältnisse mit Eisessig übergossen und einige Zeit (meist genügt eine halbe Stunde) am Rückflußkühler erhitzt. Das Kondensationsprodukt krystallisiert häufig schon während des Erhitzens oder beim Erkalten des Gemisches aus oder wird durch Zusatz von Wasser zur Abscheidung gebracht. Die Reinigung geschieht durch Umkrystallisieren aus siedendem Alkohol oder Aceton.

\section{$\beta$-m-Nitrobenzyliden-»-Phenylrhodaninsäure ${ }^{1}$.}

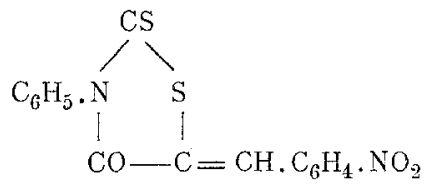

Der Körper scheidet sich nach kurzem Erhitzen von Phenylrhodaninsäure mit $m$-Nitrobenzaldehyd in Eisessig als schweres Krystallpulver ab. Die mit Alkohol gewaschenen Krystalle zeigen unter dem Mikroskope gezähnte, unregelmäßige Platten von hochgelber Farbe; nach dem Umkrystallisieren aus Alkohol erhält man kleine Schuppen von der Farbe des neutralen Kaliumchromates und schwach blauer Oberflächenfarbe. Der Körper löst sich in heißem Alkohol und Äther, schwer in Eisessig, ziemlich leicht in Aceton; der Schmelzpunkt liegt bei $240^{\circ}$ (unkorrigiert).

1 Bezüglich der Bezeichnungsweise wird auf das in der ersten Mitteilung Angeführte (1. c., p. 500) verwiesen. 
Analyse:

I. $0.2038 \mathrm{~g}$ der bei $100^{\circ}$ getrockneten Verbindung gaben $0.4188 \mathrm{~g} \mathrm{CO}$ und $0.0567 \mathrm{~g} \mathrm{H}_{2} \mathrm{O}$, entsprechend $0.1142 \mathrm{~g} \mathrm{C}$ und $0.006339 \mathrm{~g} \mathrm{H}$,

II. $0.2106 \mathrm{~g}$ Substanz gaben $16 \mathrm{~cm}^{3}$ trockenen Stickstoff bei $21^{\circ} \mathrm{C}$. und $718 m m$ Druck, entsprechend $0.017556 \mathrm{~g}$.

In 100 Teilen:

\begin{tabular}{|c|c|c|c|}
\hline & Berechnet für & & \\
\hline & $\underbrace{\mathrm{C}_{16} \mathrm{H}_{10} \mathrm{O}_{3} \mathrm{~N}_{2} \mathrm{~S}_{9}}$ & I. & II. \\
\hline C. & $56 \cdot 09$ & $56 \cdot 04$ & - \\
\hline H..... & $2 \cdot 94$ & $3 \cdot 11$ & - \\
\hline $\mathrm{N}$. & $8 \cdot 20$ & - & $8 \cdot 33$ \\
\hline
\end{tabular}

Der Schwefel wurde hier wie bei den folgenden Kondensationsprodukten nur qualitativ nachgewiesen.

$\beta$-m-Nitrobenzyliden-y-Allylrhodaninsäure.<smiles>CCCCCC=C1N[Si]SC(=O)O1</smiles>

Dieser Körper wurde noch nach dem früher angegebenen Kondensationsverfahren mittels Natronlauge in mäßiger Ausbeute gewonnen; er bildete ein hochgelbes, krystallinisches unter dem Mikroskope aus wetzsteinförmigen, zu Garben vereinigten Platten bestehendes Pulver, das in Aceton, Äther und Eisessig ziemlich leicht, verhältnismäßig schwer in Alkohol löslich war und einen Schmelzpunkt von $145^{\circ} \mathrm{C}$. aufwies.

Analyse:

I. $0.1324 \mathrm{~g}$ Substanz gaben $0.2469 \mathrm{~g} \mathrm{CO}_{2}$ und $0.041 \mathrm{~g} \mathrm{H}_{2} \mathrm{O}$, entsprechend $0.06733 \mathrm{~g} \mathrm{C}$ und $0.00458 \mathrm{~g} \mathrm{H}$.

II. $0.1538 \mathrm{~g}$ Substanz gaben $13 \mathrm{~cm}^{3}$ trockenen $\mathrm{N}$ bei $20^{\circ} \mathrm{C}$. und $715.3 \mathrm{~mm}$ Druck, entsprechend $0.01426 \mathrm{~g} \mathrm{~N}$.

In 100 Teilen:

\begin{tabular}{|c|c|c|c|}
\hline & Berechnet für & Get & \\
\hline & $\underbrace{\mathrm{C}_{13} \mathrm{H}_{10} \mathrm{O}_{3} \mathrm{~N}_{2} \mathrm{~S}_{2}}$ & I. & II. \\
\hline C & $50 \cdot 83$ & $50 \cdot 85$ & - \\
\hline $\mathrm{H} \ldots \ldots$ & $3 \cdot 29$ & $3 \cdot 46$ & - \\
\hline$N \ldots \ldots$ & $9 \cdot 16$ & - & $9 \cdot 27$ \\
\hline
\end{tabular}


$\beta-p-$ Nitrobenzyliden-y-Phenylrhodaninsäure.<smiles>O=[N+]([O-])c1ccccc1</smiles>

Die Kondensation erfolgt schon nach wenigen Minuten langem Kochen und kann durch viertelstündiges Erhitzen im Paraffinbade (wegen des starken Stoßens) zu Ende geführt werden. Man erhält nach dem Umkrystallisieren aus Alkohol feine hochgelbe Nädelchen, die sich in Aceton in der Kälte sehr wenig, in der Wärme etwas leichter lösen, auch von heißem Alkohol nur wenig aufgenommen werden und sich bei $240^{\circ} \mathrm{C}$. $z u$ schwärzen beginnen, ohne einen eigentlichen Schmelzpunkt zu zeigen. Die Ausbeute betrug hier nur $60 \%$ der Theorie.

Analyse:

I. $0.2007 \mathrm{~g}$ Substanz gaben $0.4126 \mathrm{~g} \mathrm{CO}_{2}$ und $0.057 \mathrm{~g} \mathrm{H} \mathrm{H}_{2} \mathrm{O}$, entsprechend $0.11252 \mathrm{~g} \mathrm{C}$ und $0.006373 \mathrm{~g} \mathrm{H}$.

II. $0 \cdot 216 \mathrm{~g}$ Substanz gaben $16 \cdot 2 \mathrm{~cm}^{3}$ trockenen $\mathrm{N}$ bei $19^{\circ} \mathrm{C}$. und $717 \cdot 1 \mathrm{~mm}$ Druck, entsprechend $0 \cdot 0179 \mathrm{~g} \mathrm{~N}$.

In 100 Teilen:

\begin{tabular}{|c|c|c|c|}
\hline & Berechnet für & $\mathrm{Ge}$ & den \\
\hline & $\underbrace{\mathrm{C}_{16} \mathrm{H}_{10} \mathrm{O}_{3} \mathrm{~N}_{2} \mathrm{~S}_{2}}$ & I. & II. \\
\hline$\ldots$ & $56 \cdot 09$ & $56 \cdot 06$ & - \\
\hline $\mathrm{H} \ldots \ldots$ & $2 \cdot 94$ & $3 \cdot 17$ & - \\
\hline $\mathrm{N} \ldots \ldots$ & $8 \cdot 20$ & - & $8 \cdot 28$ \\
\hline
\end{tabular}

\section{$\beta-p$-Nitrobenzyliden-v-Allylrhodaninsäure.}<smiles>O=[N+]([O-])c1ccccc1</smiles>

Die Eisessiglösung der Komponenten erstarrt beim Erhitzen zu einem festen Krystallkuchen, der, mit etwas Alkohol ausgekocht, das Kondensationsprodukt in analysenreinem Zustande hinterläßt. Es bildet dem Helianthin ähnliche, goldgelbe 
Schuppen, die sich in heißem Aceton und Eisessig lösen, schwer dagegen in Alkohol, am schwersten in Äther löslich sind. Die Verbindung, welche in einer Ausbeute von mehr als $90 \%$ der Theorie erhalten wird, schmilzt bei $153^{\circ} \mathrm{C}$. Analyse:

I. $0.2084 \mathrm{~g}$ Substanz gaben $0.3882 \mathrm{~g} \mathrm{CO}_{2}$ und $0.0635 \mathrm{~g} \mathrm{H}_{2} \mathrm{O}$, entsprechend $0 \cdot 10585 \mathrm{~g} \mathrm{C}$ und $0.0071 \mathrm{~g} \mathrm{H}$.

II. $0.1979 \mathrm{~g}$ Substanz gaben $16.8 \mathrm{~cm}^{3}$ trockenen $\mathrm{N}$ bei $21^{\circ} \mathrm{C}$. und $715 \mathrm{~mm}$ Druck, entsprechend $0.01836 g \mathrm{~N}$.

In 100 Teilen:

$\begin{array}{cccc}\mathrm{C} \ldots \ldots \ldots & \underbrace{\mathrm{C}_{13} \mathrm{H}_{10} \mathrm{O}_{3} \mathrm{~N}_{2} \mathrm{~S}_{2}}_{50 \cdot 93} & \overbrace{\text { I. }}^{\text {Gefunden }} & \text { II. } \\ \mathrm{H} \ldots \ldots & 3 \cdot 29 & 3 \cdot 79 & - \\ \mathrm{N} \ldots \ldots & 9 \cdot 16 & - & 9 \cdot 27\end{array}$

$\beta-p-O x y-m$-Methoxylbenzyliden-»-Phenylrhodaninsäure.

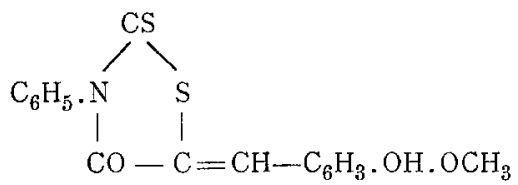

Diese aus Phenylrhodaninsäure und Vanillin erhaltene Verbindung bildet äußerst feine, hochgelbe Nadeln, welche zu einem zusammenhängenden Krystallfilz eintrocken; dieselben lösen sich in den genannten Lösungsmitteln besonders in der Wärme und schmelzen glatt bei $193^{\circ} \mathrm{C}$.

Analyse:

I. $0.2342 g$ Substanz gaben $0.510 \mathrm{~g} \mathrm{CO}_{2}$ und $0.0822 g \mathrm{H}_{2} \mathrm{O}$, entsprechend $0.1391 \mathrm{~g} \mathrm{C}$ und $0.0012 \mathrm{~g} \mathrm{H}$.

II. $0 \cdot 2574 \mathrm{~g}$ Substanz gaben $9 \cdot 8 \mathrm{~cm}^{3}$ trockenen $\mathrm{N}$ bei $20^{\circ} \mathrm{C}$. und $724 \cdot 8 \mathrm{~mm}$ Druck, entsprechend $0.01089 \mathrm{~g} \mathrm{~N}$.

In 100 Teilen:

\begin{tabular}{|c|c|c|c|}
\hline \multirow{2}{*}{\multicolumn{2}{|c|}{$\begin{array}{l}\text { Berechnet für } \\
\mathrm{C}_{17} \mathrm{H}_{13} \mathrm{O}_{3} \mathrm{NS}_{2}\end{array}$}} & \multicolumn{2}{|c|}{ Gefunden } \\
\hline & & I. & II. \\
\hline 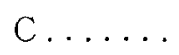 & $59 \cdot 43$ & $59 \cdot 38$ & - \\
\hline 1. & $3 \cdot 83$ & $3 \cdot 92$ & 一 \\
\hline & $4 \cdot 08$ & - & $4 \cdot 23$ \\
\hline
\end{tabular}




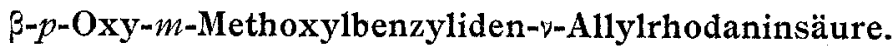

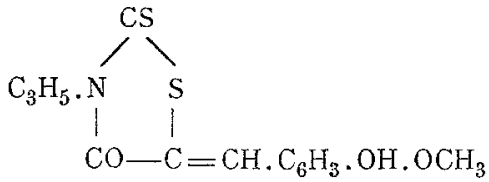

Krystallisiert aus Alkohol in kugeligen Drusen feiner orangegelber Nadeln, die sich ziemlich leicht in Aceton, Äther und Eisessig, schwer in Alkohol lösen und glatt bei $146^{\circ}$ schmelzen.

Analyse:

I. $0.2123 \mathrm{~g}$ Substanz gaben $0.4246 \mathrm{~g} \mathrm{CO}_{2}$ und $0.0846 \mathrm{~g} \mathrm{H}_{2} \mathrm{O}$, entsprechend $0 \cdot 1158 \mathrm{~g} \mathrm{C}$ und $0 \cdot 0095 \mathrm{~g} \mathrm{H}$.

II. $0 \cdot 2918 \mathrm{~g}$ Substanz gaben $12 \cdot 6 \mathrm{~cm}^{3}$ trockenen $\mathrm{N}$ bei $21^{\circ} \mathrm{C}$. und $715 \cdot 8 \mathrm{~mm}$ Druck, entsprechend $0.0138 \mathrm{~g} \mathrm{~N}$.

In 100 Teilen:

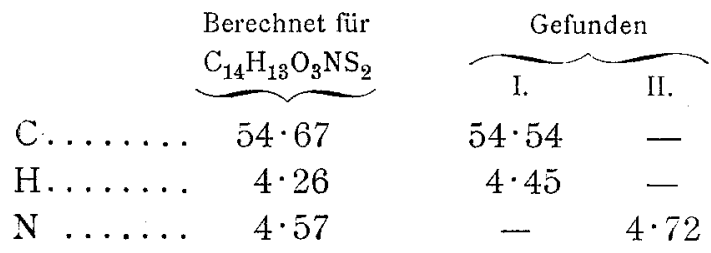

Um auch von einem der Kondensationsprodukte die Molekulargröße festzustellen, wurde die Molekulargewichtsbestimmung nach der kryoskopischen Methode bei dieser Verbindung ausgeführt.

$0 \cdot 141 \mathrm{~g}$ Substanz brachten beim Auflösen in $26.234 \mathrm{~g}$ Eisessig eine Gefrierpunktserniedrigung von $0.068^{\circ} \mathrm{C}$. hervor.

Für die Formel $\mathrm{C}_{14} \mathrm{H}_{13} \mathrm{O}_{3} \mathrm{NS}_{2}$ :

Molekulargewicht....... $\frac{\text { Berechnet }_{304 \cdot 97}^{\text {Gefunden }}}{308 \cdot 26}$ 


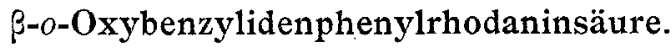

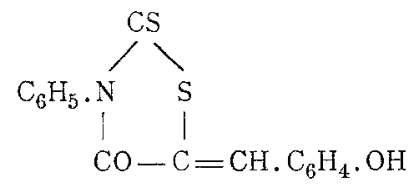

Das Kondensationsprodukt von Phenylrhodaninsäure mit Salizylaldehyd, dessen Darstellung früher (1. c. p. 509) Schwierigkeiten bereitete, wird am besten beim Erhitzen der Eisessiglösung beider Komponenten ohne Zusatz von Natriumacetat erhalten. Bei Zusatz von wasserfreiem Natriumacetat wurde meist ein farbloser Körper erhalten, der offenbar das Acetylierungsprodukt der Verbindung darstellt.

Die aus Alkohol umkrystallisierte Verbindung bildet chromgelbe Nadeln, welche leicht von Alkohol, Aceton und Eisessig in der Wärme, schwer dagegen von Äther aufgenommen werden. Der Schmelzpunkt liegt bei $172^{\circ} \mathrm{C}$.

Alkalien lösen die Verbindung mit prächtig roter Farbe, auf Zusatz von Säure wird die Verbindung wieder unverändert gefällt. Der Farbenumschlag in Gelb ist dabei auffallend scharf, so daß man die Substanz als Indikator verwenden könnte. Wolle und Seide werden von der Verbindung lebhaft gelb gefärbt; die Färbung ist ziemlich licht- und seifenecht.

Analyse:

I. $0.2076 \mathrm{~g}$ Substanz gaben $0.4665 \mathrm{~g} \mathrm{CO}_{2}$ und $0.0675 \mathrm{~g} \mathrm{H}_{2} \mathrm{O}$, entsprechend $0.1272 \mathrm{~g} \mathrm{C}$ und $0.0075 \mathrm{~g} \mathrm{H}$.

II. $0 \cdot 2508 \mathrm{~g}$ Substanz gaben $10.6 \mathrm{~cm}^{3}$ trockenen $\mathrm{N}$ bei $22^{\circ} \mathrm{C}$. und $720 \cdot 1 \mathrm{~mm}$ Druck, entsprechend $0.01163 \mathrm{~g} \mathrm{~N}$.

In 100 Teilen:

\begin{tabular}{|c|c|c|c|}
\hline \multirow{2}{*}{\multicolumn{2}{|c|}{$\begin{array}{l}\text { Berechnet für } \\
\mathrm{C}_{16} \mathrm{H}_{11} \mathrm{O}_{2} \mathrm{NS}_{2}\end{array}$}} & \multicolumn{2}{|c|}{ Gefunden } \\
\hline & & I. & II. \\
\hline & $61 \cdot 29$ & $61 \cdot 27$ & - \\
\hline & $3 \cdot 54$ & $3 \cdot 63$ & - \\
\hline & $4 \cdot 48$ & - & $4 \cdot 63$ \\
\hline
\end{tabular}

Das oben erwähnte Acetylierungsprodukt bildet nach dem Unkrystallisieren aus Alkohol feine weiße Nadeln vom Schmelz- 
punkte $202^{\circ} \mathrm{C}$. Die Analyse ergab für eine Acetyloxybenzylidenphenylrhodaninsäure stimmende Werte (gefunden $60 \cdot 67 \% \mathrm{C}$, $3 \cdot 78 \% \mathrm{H}$, berechnet für $\mathrm{C}_{18} \mathrm{H}_{13} \mathrm{O}_{3} \mathrm{NS}_{2} \quad 60 \cdot 80 \% \mathrm{C}$ und $3 \cdot 69 \% \mathrm{H})$.

Der Körper wurde nicht weiter untersucht.

Wir haben in der ersten Mitteilung eines Körpers erwähnt, der beim Kochen der Phenylrhodaninsäure mit Alkalien oder Barytwasser entsteht und in kleinen Mengen mit den Wasserdämpfen übergeht. In größerer Menge erhält man den Körper am besten durch kurzes Kochen der Phenylrhodaninsäure mit Natronlauge, Ansäuern der abgekühlten Flüssigkeit mit Salzsäure, wobei starke Entwicklung von Schwefelwasserstoff eintritt, und Umkrystallisieren der ausgeschiedenen Krystallmasse aus siedendem Alkohol. Der Körper schmilzt, wie schon angegeben, bei $151^{\circ} \mathrm{C}$, bildet große farblose Blätter, schwärzt beim Erwärmen alkalische Bleilösung, löst sich in starker Lauge auf und wird durch Säure daraus wieder gefällt. Die Analyse wies aus, daß symmetrischer Diphenylthioharnstoff vorlag.

Analyse:

$0.202 \mathrm{~g}$ Substanz gaben $0.5056 \mathrm{~g} \mathrm{CO}_{2}$ und $0.097 \mathrm{~g} \mathrm{H}_{2} \mathrm{O}$, entsprechend $0 \cdot 13787 \mathrm{~g} \mathrm{C}$ und $0.0108 \mathrm{~g} \mathrm{H}$.

In 100 Teilen:

\begin{tabular}{|c|c|c|}
\hline & $\begin{array}{c}\text { Berechnet für } \\
\mathrm{C}_{13} \mathrm{H}_{12} \mathrm{~N}_{2} \mathrm{~S}\end{array}$ & Gefunden \\
\hline & $68 \cdot 42$ & $68 \cdot 25$ \\
\hline 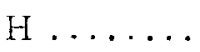 & $5 \cdot 26$ & $5 \cdot 36$ \\
\hline
\end{tabular}

In der Flüssigkeit, aus welcher der Körper ausgefällt wurde, lassen sich reichliche Mengen von Thioglykolsäure durch die bekannte Eisenreaktion nachweisen, so daß der Zerfall der Phenylrhodaninsäure unter dem hydrolysierenden Einfluß der Basen durch die Gleichung:

$2 \mathrm{C}_{9} \mathrm{H}_{7} \mathrm{ONS}_{2}+4 \mathrm{H}_{2} \mathrm{O}=2 \mathrm{C}_{2} \mathrm{H}_{4} \mathrm{SO}_{2}+\mathrm{SH}_{2}+\mathrm{CO}_{2}+\mathrm{CS}\left(\mathrm{NHC}_{6} \mathrm{H}_{5}\right)_{2}$

wiedergegeben werden kann; möglicherweise erfolgt zunächst eine Spaltung in Thioglykolsäure und Phenylsenföl, von dem 
ein Teil in Kohlendioxyd, Schwefelwasserstoff und Anilin zerfällt, welches mit einem zweiten Molekül Senföl zu dem symmetrischen Diphenylthioharnstoff zusammentritt.

Auch die Allylrhodaninsäure wird durch Basen in Kohlendioxyd, Schwefelwasserstoff, Thioglykolsäure und wahrscheinlich Diallylthioharnstoff zerlegt, welch letzterer aber nur in Gestalt eines alkalische Bleilösung schwärzenden Öls erhalten wurde.

v-Methylrhodaninsäure.<smiles>CN1SSCC1=O</smiles>

Um die von uns aufgefundene Reaktion auf ihre allgemeine Giltigkeit zu prüfen, wurde der Versuch auch mit Thioglykolsäure und Methylsenföl durchgeführt.

Man versetzt zu diesem Zwecke das Methylsenföl mit etwas mehr als der theoretischen Menge Thioglykolsäure in wässeriger Lösung, fügt dem Gemenge zur Erhöhung der Löslichkeit des Senföles etwas Alkohol zu und erhitzt längere Zeit am Rückflußkühler zum Sieden. Nach einiger Zeit wird die Flüssigkeit trübe; läßt man dann erkalten, so erstarrt das am Boden des Kolbens befindliche ölige Liquidum zu einem festen Krystallkuchen, indem gleichzeitig die Flüssigkeit sich mit feinen, dünnen, seideglänzenden Nadeln von mehreren Zentimetern Länge füllt.

Der durch Krystallisation aus Alkohol eventuell unter Zusatz von Tierkohle erhaltene reine Körper bildet breite, dünne, lange Nadeln oder Platten von schwach grünlichgelber Farbe; auch aus viel siedendem Wasser kann die Verbindung umkrystallisiert werden, wobei die Flüssigkeit beim Erkalten zunächst milchig wird und sich dann in ein Haufwerk feiner, fast weißer Nadeln verwandelt. 
Der Körper besitzt neutrale Eigenschaften, ${ }^{1}$ ist in Alkohol, Äther, Aceton und Eisessig bereits in der Kälte ziemlich leicht löslich, schmilzt glatt bei $72^{\circ} \mathrm{C}$. und besitzt die Zusammensetzung der erwarteten Methylrhodaninsäure, die sich nach folgendem Schema gebildet hat:<smiles>CN=[N+]=S</smiles>

Analyse:

1. $0.216 \mathrm{~g}$ exsikkatortrockene Substanz gaben $0.2578 \mathrm{~g} \mathrm{CO}_{2}$ und $0.069 \mathrm{z} \mathrm{g}$ $\mathrm{H}_{2} \mathrm{O}$, entsprechend $0.0703 \mathrm{~g} \mathrm{C}$ und $0.00774 \mathrm{~g} \mathrm{H}$.

II. $0 \cdot 1971 \mathrm{~g}$ Substanz gaben $17 \cdot 2 \mathrm{~cm}^{3}$ trockenen $\mathrm{N}$ bei $20^{\circ} \mathrm{C}$. und $727.5 \mathrm{~mm}$ Druck, entsprechend $0.01919 \mathrm{~g} \mathrm{~N}$.

III. $0.1912 \mathrm{~g}$ Substanz gaben beim Schmelzen mit Natriumkarbonat und Kaliumchlorat etc. $0 \cdot 6048 \mathrm{~g} \mathrm{BaSO}_{4}$, entsprechend $0.08299 \mathrm{~g} \mathrm{~S}$.

In 100 Teilen:

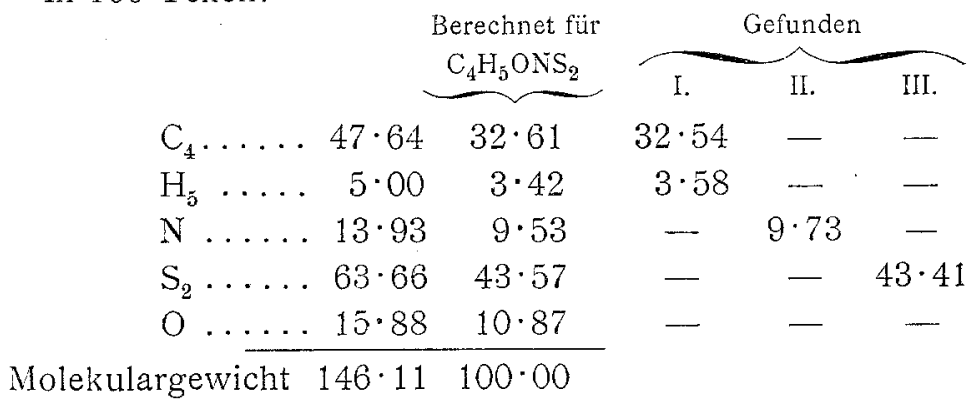

Zur Feststellung der Molekulargröße wurde eine Molekulargewichtsbestimmung nach der kryoskopischen Methode mit Eisessig als Lösungsmittel ausgeführt.

$0.2019 \mathrm{~g}$ Substanz bewirkten beim Auflösen in $15.29 \mathrm{~g}$ Eisessig eine Gefrierpunktserniedrigung von $0.336^{\circ} \mathrm{C}$.

I Der Name Rhodanins äure paßt eigentlich auf alle hier beschriebenen Körper nicht mehr, da durch den Eintritt der substituierenden Gruppe die schwach sauren Eigenschaften der Rhodaninsäure aufgehoben sind; vielleicht könnte man sich mit dem Namen $\gg$ Rhodanin begnïgen. 
Für die Formel $\mathrm{C}_{4} \mathrm{H}_{5} \mathrm{ONS}_{2}$ :

Molekulargewicht....... $\frac{\underbrace{\text { Berechnet }}_{146 \cdot 11}}{153 \cdot 27}$ Gefunden

Die Methylrhodaninsäure ist mit der von Berlinerblau ${ }^{1}$ aus $\alpha$-Brompropionsäure und Rhodanammonium dargestellten a-Rhodaninpropionsäure<smiles>CC1O[SiH2]N[SiH2]1</smiles>

der nach unserer Bezeichung der Name $\beta$-Methylrhodaninsäure zukommen müßte, isomer.

Die beschriebene Methylrhodaninsäure gibt wie die anderen Rhodaninsäuren mit aromatischen Aldehyden auf das leichteste Kondensationsprodukte, die schon beim kurzen Erhitzen der Komponenten in eisessigsaurer Lösung entstehen und, soweit bekannt, ebenfalls stark gelb gefärbte Körper von großem Krystallisationsvermögen sind.

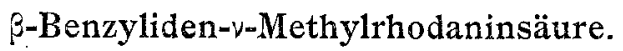<smiles>CCCCCCCC=C1C=C2ON(C)[SH]2S1</smiles>

Der Körper bildet, aus Alkohol krystallisiert, eine aus lockeren, wolligen Nadeln bestehende Krystallmasse von schwefelgelber Farbe und dem Schmelzpunkte $169^{\circ} \mathrm{C}$. Die Substanz ist in Aceton, Eisessig und Alkohol in der Kälte wenig, in der Wärme leichter löslich, wenig wird sie von Äther aufgenommen.

Analyse:

I. $0.2082 g$ Substanz gaben $0.4275 \mathrm{~g} \mathrm{CO}_{2}$ und $0.0751 \mathrm{~g} \mathrm{H}_{2} \mathrm{O}$, entsprechend $0.1166 \mathrm{~g} \mathrm{C}$ und $0.0084 \mathrm{~g} \mathrm{H}$.

II. $0 \cdot 2512 \mathrm{~g}$ Substanz gaben $14 \mathrm{~cm}^{3}$ trockenen $\mathrm{N}$ bei $20^{\circ} \mathrm{C}$. und $720 \cdot 1 \mathrm{~mm}$ Druck, entsprechend $0.01546 \mathrm{~g} \mathrm{~N}$.

1 Berichte der Deutschen chem. Gesellsch., 19, 125. 
In 100 Teilen:

\begin{tabular}{|c|c|c|c|}
\hline & Berechnet für & Gef & \\
\hline & $\underbrace{\mathrm{C}_{11} \mathrm{H}_{9} \mathrm{ONS}_{2}}$. & I. & II. \\
\hline C & $56 \cdot 13$ & $55 \cdot 99$ & - \\
\hline H. & $3 \cdot 85$ & $4 \cdot 03$ & - \\
\hline$N$ & $5 \cdot 96$ & - & $6 \cdot 15$ \\
\hline
\end{tabular}

\section{$\beta$ - $p$-Methoxylbenzyliden- $\nu$-Methylrhodaninsäure.}<smiles></smiles>

Dieser aus Methylrhodaninsäure und Anisaldehyd gewonenne Körper stellt feine, goldgelbe Nadeln vom Schmelzpunkte $181^{\circ} \mathrm{C}$. dar, die einen zusammenhängenden Krystallfilz bilden; dieselben lösen sich in Aceton ziemlich leicht schon in der Kälte, in Alkohol und Eisessig leichter in der Wärme, wenig in Äther.

Analyse:

$0.2267 \mathrm{~g}$ Substanz gaben $0.4503 \mathrm{~g} \mathrm{CO}_{2}$ und $0.0879 \mathrm{~g} \mathrm{H}_{2} \mathrm{O}$, entsprechend $0 \cdot 1228 \mathrm{~g} \mathrm{C}$ und $0.0098 \mathrm{~g} \mathrm{H}$.

In 100 Teilen:
Berechnet für $\mathrm{C}_{12} \mathrm{H}_{11} \mathrm{O}_{2} \mathrm{NS}_{2}$
C....... $54 \cdot 28$
Gefunden
H $\ldots \ldots \ldots \quad 4 \cdot 17$
$54 \cdot 16$
$4 \cdot 33$

$\beta-m$-Nitrobenzyliden-y-Methylrhodaninsäure.<smiles>CN1OC2=CC(=O)OC1S2</smiles>

Diese aus Methylrhodaninsäure und $m$-Nitrobenzaldehyd dargestellte, durch Umkrystallisieren aus Aikohol gereinigte Substanz bildet grünlichgelbe Nadeln vom Schmelzpunkte 
$233^{\circ} \mathrm{C}$, die sich in den schon mehrfach genannten organischen Lösungsmitteln in der Kälte wenig, in der Wärme leicht lösen. Analyse:

$0.2296 \mathrm{~g}$ Substanz gaben $0.3969 \mathrm{~g} \mathrm{CO}_{2}$ und $0.0638 \mathrm{~g} \mathrm{H}_{2} \mathrm{O}$, entsprechend $0 \cdot 1082 \mathrm{~g} \mathrm{C}$ und $0.0071 \mathrm{~g} \mathrm{H}$.

In 100 Teilen:

\begin{tabular}{|c|c|c|}
\hline & $\begin{array}{l}\text { Berechnet für } \\
\mathrm{C}_{11} \mathrm{H}_{8} \mathrm{O}_{3} \mathrm{~N}_{2} \mathrm{~S}_{2}\end{array}$ & Gefunden \\
\hline & $47 \cdot 10$ & $47 \cdot 14$ \\
\hline & $2 \cdot 88$ & $3 \cdot 10$ \\
\hline
\end{tabular}

$\beta-p$-Nitrobenzyliden-y-Methylrhodaniṇsäure.<smiles>CN1OC2=CC(=O)OCS[SH]21</smiles>

Der Körper bildet orangegelbe Schüppchen, die in Äther auch in der Wärme wenig löslich sind, aber von Alkohol, Aceton und Eisessig in der Siedehitze leicht aufgenommen werden und bei $205^{\circ} \mathrm{C}$. schmelzen.

Analyse:

$0.2044 \mathrm{~g}$ Substanz gaben $0.3527 \mathrm{~g} \mathrm{CO}_{2}$ und $0.0557 \mathrm{~g} \mathrm{H}_{2} \mathrm{O}$, entsprechend $0.09618 \mathrm{~g} \mathrm{C}$ und $0.0062 \mathrm{~g} \mathrm{H}$.

In 100 Teilen:

\begin{tabular}{|c|c|c|}
\hline & $\begin{array}{l}\text { Berechnet für } \\
\mathrm{C}_{11} \mathrm{H}_{8} \mathrm{O}_{3} \mathrm{~N}_{2} \mathrm{~S}_{2}\end{array}$ & Gefunden \\
\hline & $47 \cdot 10$ & $47 \cdot 05$ \\
\hline 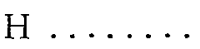 & $2 \cdot 88$ & $3 \cdot 04$ \\
\hline
\end{tabular}

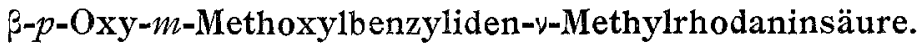<smiles>COOc1ccccc1</smiles>

Dieser aus Methylrhodaninsäure und Vanillin erhältliche Körper bildet orangegelbe Nadeln, die zum Teile garbenförmig 
zusammengelegt sind und bei $199^{\circ} \mathrm{C}$. schmelzen. Die Löslichkeitsverhältnisse sind dieselben wie die der vorstehend beschriebenen Verbindung.

Analyse:

$0.2057 \mathrm{~g}$ Substanz gaben $0.3861 \mathrm{~g} \mathrm{CO}_{2}$ und $0.0747 \mathrm{~g}$. $\mathrm{H}_{2} \mathrm{O}$, entsprechend $0.1053 \mathrm{~g}$ C und $0.00835 \mathrm{~g} \mathrm{H}$.

In 100 Teilen:

$\begin{array}{ccc}\text { C } \ldots \ldots \ldots & \begin{array}{r}\text { Berechnet für } \\ \mathrm{C}_{12} \mathrm{H}_{11} \mathrm{O}_{3} \mathrm{NS}_{2}\end{array}\end{array} \quad \underbrace{\text { Gefunden }}_{51 \cdot 20}$

ß-Methylen-3, 4-Dioxybenzyliden-y-Methylrhodaninsäure.<smiles>COc1ccccc1</smiles>

Das Kondensationsprodulkt aus Methylrhodaninsäure und Piperonal bildet hochgelbe, mikroskopische Nadeln (aus Alkohol) vom Schmelzpunkte $204^{\circ} \mathrm{C}$, die sich in Äther und Alkohol in der Kälte sehr wenig lösen, in der Wärme auch von Aceton und Eisessig leicht aufgenommen werden.

Analyse:

$0.201 \mathrm{~g}$ Substanz gaben $0.3792 \mathrm{~g} \mathrm{CO}_{2}$ und $0.0621 \mathrm{~g} \mathrm{H}_{2} \mathrm{O}$, entsprechend $0.1034 g \mathrm{C}$ und $0.00694 \mathrm{~g} \mathrm{H}$.

In 100 Teilen:

\begin{tabular}{|c|c|c|}
\hline & $\begin{array}{l}\text { Berechnet für } \\
\mathrm{C}_{12} \mathrm{H}_{9} \mathrm{O}_{3} \mathrm{NS}_{2}\end{array}$ & Gefunden \\
\hline & $51 \cdot 57$ & $51 \cdot 44$ \\
\hline & $3 \cdot 25$ & $3 \cdot 45$ \\
\hline
\end{tabular}

$\beta$-Cinnamylen-y-Methylrhodaninsäure.<smiles>CN1[C]2SC(=CC=Cc3ccccc3)O[As]21</smiles>

Das Kondensationsprodukt mit Zimtaldehyd stellt dunkelorangegelbe Nadeln vom Schmelzpunkte $226^{\circ} \mathrm{C}$. dar, welche 
ähnliche Lösungsverhältnisse zeigen, wie die oben beschriebenen Körper, nur daß sie in Alkohol bereits in der Kälte ziemlich leicht löslich sind.

Analyse:

$0.2575 \mathrm{~g}$ Substanz gaben $0.5622 \mathrm{~g} \mathrm{CO}_{2}$ und $0.0995 \mathrm{~g} \mathrm{H}_{2} \mathrm{O}$, entsprechend $0.1533 \mathrm{~g} \mathrm{C}$ und $0.1112 \mathrm{~g} \mathrm{H}$.

In 100 Teilen:

$$
\begin{aligned}
& \text { Berechnet für } \\
& \text { C....... } \frac{\mathrm{C}_{13} \mathrm{H}_{11} \mathrm{ONS}_{2}}{59 \cdot 71} \quad \frac{\text { Gefunden }}{59 \cdot 53} \\
& \text { H ..... } 4 \cdot 25 \quad 4 \cdot 32
\end{aligned}
$$

ע-Äthylrhodaninsäure.<smiles>CCN1[CH]SC1=O</smiles>

Wird Äthylsenföl mit etwas mehr als der theoretischen Menge von Thioglykolsäure mit stark verdünntem Alkohol gekocht, so sinkt das anfangs obenauf schwimmende Öl unter sichtlicher Volumsvermehrung bald unter. Nachdem der Senfölgeruch fast verschwunden ist, wird etwas unangegriffenes Senföl unter Durchleiten von Wasserdampf übergetrieben und das ölige Liquidum im Scheidetrichter von der Flüssigkeit, die überschüssige Thioglykolsäure enthält, getrennt, eventuell zur Reinigung mehrere Male mit Wasser, dem man anfangs etwas Sodalösung zusetzt, gewaschen und im Exsikkator getrocknet.

Wie bei der Allylrhodaninsäure gelang es auch hier nicht, das Ö1 zum Erstarren zu bringen. Daß aber wirklich die nach dem Schema

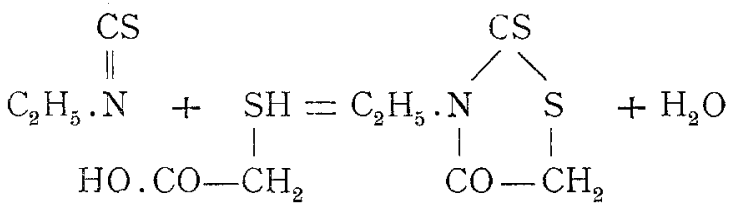

entstandene $\nu$-Äthylrhodaninsäure vorlag, ergibt die Analyse der unten beschriebenen Kondensationsprodukte, besonders 
des mit $m$-Nitrobenzaldehyds. Die Kondensationsprodukte der Äthylrhodaninsäure zeichnen sich durch besondere Krystalli sationsfähigkeit und äußerst prächtige Färbung aus.

\section{$\beta$-Benzyliden-v-Äthylrhodaninsäure.}<smiles>CCCCCCCCCCCCCCCCC</smiles>

Wird die ölige Äthylrhodaninsäure mit der einem Moleküle entsprechenden Menge Benzaldehyd und etwas Eisessig durch eine halbe Stunde am Rückflußkühler gekocht, so fällt auf Zusatz von Wasser aus der Eisessiglösung ein dickliches ÖI aus, das beim Übergießen mit Alkohol sich in einen Krystallbrei verwandelt, der am besten aus 70 prozentigem Alkohol umkrystallisiert wird. Die Benzylidenäthylrhodaninsäure bildet prächtige, lebhaft glänzende, flache Nadeln von goldgelber, etwas ins grünliche ziehender Farbe und einem Schmelzpunkte von $149^{\circ} \mathrm{C}$. Die Substanz ist in Aceton und Äther leicht, in Alkohol und Eisessig besonders in der Wärme löslich.

Analyse:

$0.2163 \mathrm{~g}$ Substanz gaben $0.4572 \mathrm{~g} \mathrm{CO}_{2}$ und $0.0878 \mathrm{~g} \mathrm{H}_{2} \mathrm{O}$, entsprechend $0.1249 \mathrm{~g} \mathrm{C}$ und $0.0098 \mathrm{~g} \mathrm{H}$.

In 100 Theilen:

\begin{tabular}{|c|c|c|}
\hline की & $\begin{array}{l}\text { Berechnet für } \\
\mathrm{C}_{12} \mathrm{H}_{11} \mathrm{ONS}_{2}\end{array}$ & Gefunden \\
\hline $\mathrm{C}$ & $57 \cdot 77$ & $57 \cdot 65$ \\
\hline 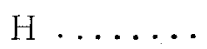 & $4 \cdot 45$ & $4 \cdot 54$ \\
\hline
\end{tabular}

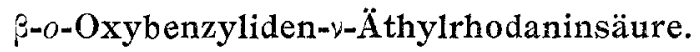

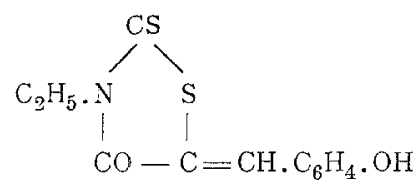

Auch bei der Kondensation von Äthylrhodaninsäure und Salizylaldehyd erhält man anfangs nur ein Öl, das erst beim Aufnehmen in Alkohol und langsamen Verdunstenlassen in äußerst prächtigen, orangegelben Nadeln anschießt. Dieselben 
erinnern durch ihre schwach blaue Oberflächenfarbe an gewisse Pikrate. Der Schmelzpunkt liegt bei $190^{\circ} \mathrm{C}$. Die Verbindung ist in Aceton und Alkohol in der Kälte schwer, in der Hitze leicht löslich, ebenso in der Wärme in Eisessig und Äther.

Analyse:

$0.160 \mathrm{~g}$ Substanz gaben $0.3175 \mathrm{~g} \mathrm{CO}_{3}$ und $0.0629 \mathrm{~g} \mathrm{H}_{2} \mathrm{O}$, entsprechend $0.0866 \mathrm{~g} \mathrm{C}$ und $0.007 \mathrm{~g} \mathrm{H}$.

In 100 Teilen:

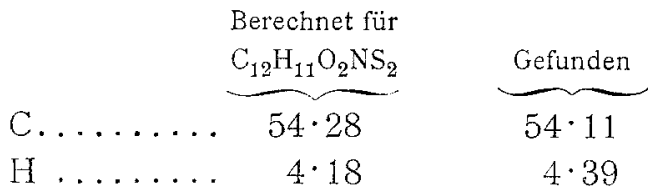

Diese durch den Besitz einer auxochromen Hydroxylgruppe ausgezeichnete Verbindung ist ein Farbstoff, der Wolle und Seide prächtig gelb färbt.

Alkalien oder Ammoniak lösen die Substanz mit rötlichgelber Farbe, auf Zusatz von Säure tritt scharfer Umschlag zu Gelb ein und die Substanz fällt unverändert in feinen Krystallnadeln aus.

ß-p-Methoxylbenzyliden-y-Äthylrhodaninsäure.<smiles>CCCC1OC(=O)N(CC)[SiH2]S1</smiles>

Die Verbindung stellt chromgelbe, sehr feine, meist zu Drusen oder einem Krystallfilz vereinigte Nadeln vom Schmelzpunkte $143^{\circ}$ dar, die in Aceton und Äther leicht, in Eisessig und Alkohol nur in der Wärme leicht löslich sind.

Analyse:

$0.2098 g$ Substanz gaben $0.430 \mathrm{~g} \mathrm{CO}_{2}$ und $0.0885 \mathrm{~g} \mathrm{H}_{2} \mathrm{O}$, entsprechend $0.1173 \mathrm{~g} \mathrm{C}$ und $0.00989 \mathrm{~g} \mathrm{H}$.

In 100 Teilen:

\begin{tabular}{|c|c|c|}
\hline & $\begin{array}{l}\text { Berechnet für } \\
\mathrm{C}_{13} \mathrm{H}_{13} \mathrm{O}_{2} \mathrm{NS}_{2}\end{array}$ & Gefunden \\
\hline & $55 \cdot 86$ & $55 \cdot 89$ \\
\hline . & $4 \cdot 69$ & $4 \cdot 71$ \\
\hline
\end{tabular}




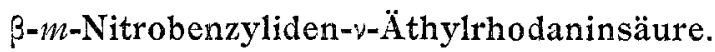<smiles>CCCCC=C1ON(CCCC)[CH-]S1</smiles>

Die Substanz bildet grünlichgelbe, lebhaft glänzende Blättchen oder flache Nadeln vom Schmelzpunkte $188^{\circ} \mathrm{C}$., die von Aceton und Eisessig schon in der Kälte, von Alkohol und Äther erst in der Wärme leichter aufgenommen werden. Dieser Körper wurde einer vollständigen Analyse unterworfen.

I. $0.209 \mathrm{~g}$ Substanz gaben $0.3748 \mathrm{~g} \mathrm{CO} \mathrm{CO}_{2}$ und $0.0677 \mathrm{~g} \mathrm{H}_{2} \mathrm{O}$, entsprechend $0.1022 \mathrm{~g} \mathrm{C}$ und $0.00757 \mathrm{~g} \mathrm{H}$.

II. $0.2037 \mathrm{~g}$ Substanz gaben $17.8 \mathrm{~cm}^{3}$ trockenen $\mathrm{N}$ bei $22^{\circ} \mathrm{C}$. und $721.5 \mathrm{~mm}$ Druck, entsprechend $0.01957 \mathrm{gN}$.

III. $0 \cdot 1685 \mathrm{~g}$ Substanz gaben nach dem Glühen mit Soda und Kaliumchlorat etc. $0.2683 \mathrm{~g} \mathrm{BaSO}_{4}$, entsprechend $0.03684 \mathrm{~g} \mathrm{~S}$.

In 100 Teilen:

\begin{tabular}{|c|c|c|c|c|}
\hline & \multirow{2}{*}{$\begin{array}{l}\text { Berechnet für } \\
\mathrm{C}_{12} \mathrm{H}_{10} \mathrm{O}_{3} \mathrm{~N}_{2} \mathrm{~S}_{2}\end{array}$} & \multicolumn{3}{|c|}{ Gefunden } \\
\hline & & I. & II. & III. \\
\hline $\mathrm{C}_{12} \ldots \ldots 142 \cdot 92$ & $48 \cdot 93$ & $48 \cdot 90$ & - & - \\
\hline $\mathrm{H}_{10} \ldots \ldots 10 \cdot 00$ & $3 \cdot 42$ & . $3 \cdot 62$ & - & - \\
\hline $\mathrm{N}_{2} \ldots \ldots 27 \cdot 86$ & $9 \cdot 54$ & 一 & $9 \cdot 61$ & - \\
\hline $\mathrm{S}_{2} \ldots \ldots 63 \cdot 66$ & $21 \cdot 80$ & - & - & $21 \cdot 87$ \\
\hline $\mathrm{O}_{3} \ldots \ldots 47 \cdot 64$ & $10 \cdot 31$ & $\ldots$ & 一 & - \\
\hline
\end{tabular}

ß-p-Oxy-m-Methoxylbenzyliden-y-Äthylrhodaninsäure.<smiles>CCCCCCCOc1ccccc1</smiles>

Dieses aus Vanillin und der Äthylrhodaninsäure hergestellte Kondensationsprodukt bildet feine, oft drusenartig vereinigte Nadeln oder auch einen hochgelben Krystallfilz; die Substanz weist einen Schmelzpunkt von $140^{\circ}$ auf und ist in den genannten Lösungsmitteln, besonders aber in Äther löslich. 
Analyse:

$0.1975 \mathrm{~g}$ Substanz gaben $0.3832 \mathrm{~g} \mathrm{CO}$ und $0.080 \mathrm{~g} \mathrm{H}_{2} \mathrm{O}$, entsprechend $0.1445 \mathrm{~g} \mathrm{C}$ und $0.0089 \mathrm{~g} \mathrm{H}$.

In 100 Teilen:

\begin{tabular}{|c|c|c|}
\hline & $\begin{array}{l}\text { Berechnet für } \\
\mathrm{C}_{13} \mathrm{H}_{13} \mathrm{O}_{3} \mathrm{NS}_{2}\end{array}$ & Gefunden \\
\hline & $52 \cdot 83$ & $52 \cdot 91$ \\
\hline 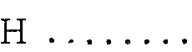 & $4 \cdot 44$ & $4 \cdot 53$ \\
\hline
\end{tabular}

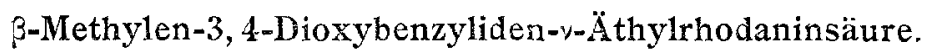

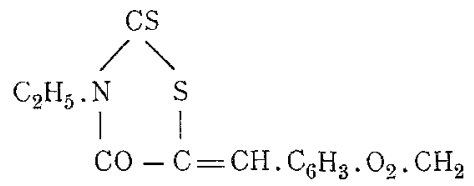

Aus Piperonal und der äthylierten Rhodaninsäure gewonnen, stellt sie wollige, hochgelbe Nadeln vom Schmelzpunkte $154^{\circ}$ dar, welche sich bei Zimmertemperatur leicht in Aceton lösen, von Alkohol, Eisessig oder Äther aber erst in der Siedehitze leichter aufgenommen werden.

Analyse:

$0.2398 \mathrm{~g}$ Substanz gaben $0.4679 \mathrm{~g} \mathrm{CO}_{2}$ und $0.0835 \mathrm{~g} \mathrm{H}_{2} \mathrm{O}$, entsprechend $0.1276 \mathrm{~g} \mathrm{C}$ und $0.0093 \mathrm{~g} \mathrm{H}$.

In 100 Teilen:

\begin{tabular}{|c|c|c|}
\hline & $\begin{array}{l}\text { Berechnet für } \\
\mathrm{C}_{13} \mathrm{H}_{11} \mathrm{O}_{3} \mathrm{NS}_{2}\end{array}$ & Gefunden \\
\hline & $53 \cdot 20$ & $53 \cdot 21$ \\
\hline & $3 \cdot 78$ & $3 \cdot 89$ \\
\hline
\end{tabular}

$\beta$-Cinnamylen-y-Äthylrhodaninsäure.<smiles>CCCCCCCC=Cc1ccccc1</smiles>

Die Substanz bildet gelbe Schüppchen, ungefähr von dem Aussehen des Mussivgoldes, zeigt einen Schmelzpunkt von 
$187^{\circ}$ C. und die gleichen Löslichkeitsverhältnisse wie die vorige Verbindung.

Analyse:

$0.2053 \mathrm{~g}$ Substanz gaben $0.4593 \mathrm{~g} \mathrm{CO}_{2}$ und $0.0894 \mathrm{~g} \mathrm{H}_{2} \mathrm{O}$, entsprechend $0.12525 \mathrm{~g} \mathrm{C}$ und $0.00999 \mathrm{~g} \mathrm{H}$.

In 100 Teilen:

$\begin{array}{ccc}\mathrm{C} \ldots \ldots \ldots & \begin{array}{r}\text { Berechnet für } \\ \mathrm{C}_{14} \mathrm{H}_{13} \mathrm{ONS}_{2}\end{array} & \underbrace{\text { Gefunden }}_{61.03} \\ \mathrm{H} \ldots \ldots \ldots & 4.76\end{array} \quad \begin{array}{r}4.01 \\ 4.87\end{array}$

$\beta$-Methyl-v-Phenylrhodaninsäure.

Alle bisher beschriebenen substituierten Rhodaninsäuren wurden durch Verbindung von Senfölen mit Thioglykolsäure dargestellt; es war nun wünschenswert, auch eine andere Thio- oder richtiger Sulfhydrylfettsäure auf das diesbezügliche Verhalten zu Senfölen zu prüfen.

Wir verwandten dazu die $\alpha$-Thiomilchsäure.

Um diesen Körper zu erhalten, wurde aus $\alpha$-Brompropionsäureäthylester und Thioharnstoff zunächst das $\beta$-Methylthiohydantoin ${ }^{1}$

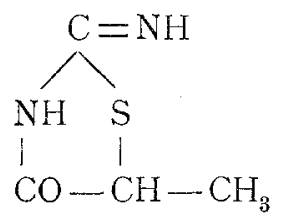

dargestellt und diese Verbindung durch Kochen mit Natronlauge in Cyanamid und $\alpha$-Thiomilchsäure gespalten. Letztere konnte der angesäuerten Lösung durch mehrmaliges Ausschütteln mit Äther entżogen werden.

Der Ätherrückstand wurde mit der äquivalenten Menge Phenylsenföl unter Zusatz von Wasser und etwas Alkohol am Rückflußkühler gekocht. Nach $1 \frac{1}{2}$ stündigem Erhitzen wurde das ausgeschiedene Öl mit Alkohol übergossen, wodurch es sofort erstarrte und dann aus einer größeren Alkoholmenge umkrystallisiert. Man erhält dünne Platten oder flache Nadeln von fast weißer, etwas ins Grünliche ziehender Farbe und

1 Dixon, Proc. chem. soc., 123, 115. 
einem Schmelzpunkte von 118 bis $119^{\circ}$ C. Die neue Substanz ist in Aceton sehr leicht, in Eisessig, Ather und Alkohol in der Kälte wenig, in der Wärme leichter löslich. Wie die Analyse ausweist, ist die Reaktion im erwarteten Sinne verlaufen:

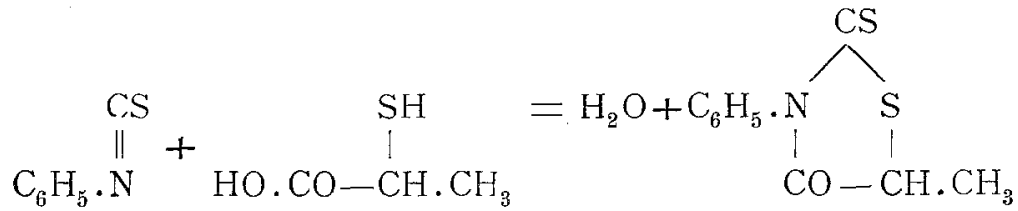

und wird man daher die neue Substanz als $\beta$-Methyl- $y$-Phenylrhodaninsäure zu bezeichnen haben.

Analyse:

I. $0.2365 \mathrm{~g}$ Substanz gaben $0.4652 \mathrm{~g} \mathrm{CO}_{2}$ und $0.0886 \mathrm{~g} \mathrm{H} \mathrm{H}_{2} \mathrm{O}$, entsprechend $0.1268 \mathrm{~g} \mathrm{C}$ und $0.0099 \mathrm{~g} \mathrm{H}$.

II. $0.2452 \mathrm{~g}$ Substanz gaben $14.2 \mathrm{~cm}^{3}$ trockenen $\mathrm{N}$ bei $22^{\circ} \mathrm{C}$. und $723.5 \mathrm{~mm}$ Druck, entsprechend $0.01566 \mathrm{~g}$ N.

III. $0 \cdot 240 \mathrm{~g}$ Substanz gaben beim Schmelzen mit Soda und Kaliumchlorat etc. $0.5011 \mathrm{~g} \mathrm{BaSO}_{4}$, entsprechend $0.0688 \mathrm{~g} \mathrm{~S}$.

In 100 Theilen:

\begin{tabular}{|c|c|c|c|c|}
\hline & Berechnet für & & efunden & \\
\hline & $\underbrace{\mathrm{C}_{10} \mathrm{H}_{9} \mathrm{ONS}_{2}}$ & I. & II. & III. \\
\hline$C_{10} \ldots \ldots 119 \cdot 10$ & $53 \cdot 76$ & $53 \cdot 64$ & - & - \\
\hline $\mathrm{H}_{9} \ldots \ldots \quad 9 \cdot 00$ & $4 \cdot 06$ & $4 \cdot 18$ & - & 一 \\
\hline$\ldots 13 \cdot 93$ & $6 \cdot 29$ & - & $6 \cdot 39$ & 一 \\
\hline$\ldots \ldots 63 \cdot 66$ & $28 \cdot 73$ & - & 一 & $28 \cdot 67$ \\
\hline$\ldots \ldots 15 \cdot 88$ & $7 \cdot 16$ & - & - & - \\
\hline
\end{tabular}

Zur Feststellung der Molekulargröße wurde eine Molekulargewichtsbestimmung nach der kryoskopischen Methode ausgeführt.

$0 \cdot 2314 g$ Substanz bewirkten beim Auflösen in $24.79 g$ Eisessig eine Gefrierpunktserniedrigung von $0.16^{\circ} \mathrm{C}$.

$$
\text { Molekulargewicht ....... } \frac{\begin{array}{c}
\text { Berechnet für } \\
\mathrm{C}_{10} \mathrm{H}_{9} \mathrm{ONS}_{2}
\end{array}}{221 \cdot 57} \quad \underbrace{\text { Gefunden }}_{227 \cdot 5}
$$

Derselbe Körper mußte sich auch nach der Methode von v. Braun aus phenyldithiokarbaminsaurem Ammonium und $\alpha$-Brompropionsäureester darstellen lassen: 
180 R. Andreaseh und A. Zipser, Substituierte Rhodaninsäuren.
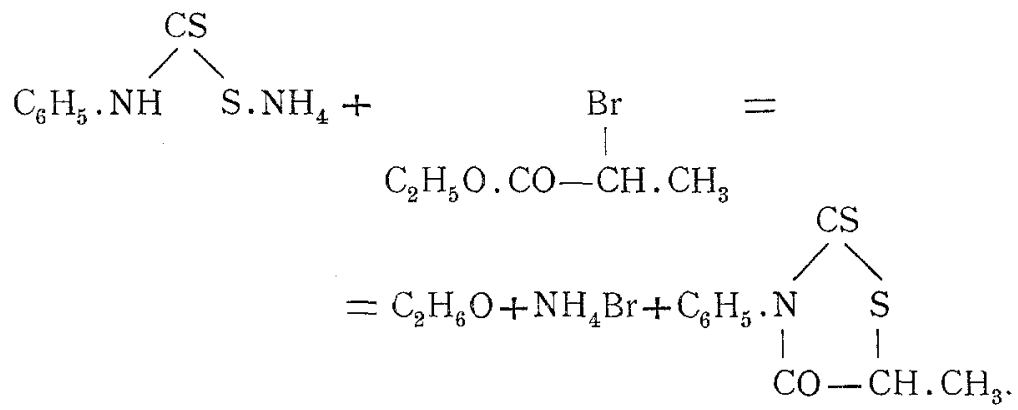

Es wurden beide Körper in molekularen Verhältnissen mit Alkohol am Rückflußkühler durch eine halbe Stunde im Sieden erhalten, darnach der Alkohol zum Teile abdestilliert und der Rückstand mit Wasser versetzt. Das sich anfangs als dickliches Öl abscheidende Einwirkungsprodukt erstarrte bald zu einem Krystallkuchen, der nach zweimaligem Umkrystallisieren den Schmelzpunkt $119^{\circ}$ aufwies und in allen Eigenschaften mit der aus Phenylsenföl und $\alpha$-Thiomilchsäure dargestellten Verbindung übereinstimmte.

Versuche, aus dieser $\beta$-Methylphenylrhodaninsäure Kondensationsprodukte mit Aldehyden darzustellen, mißlangen; es wurden stets die unveränderten Ausgangsmaterialien zurückerhalten. Es war dies vorauszusehen, da der Verbindung die zur Kondensation notwendige, zwischen den beiden negativen Gruppen $\mathrm{CO}$ und $\mathrm{S}$ stehende Methylengruppe abgeht.

Noch soll nicht unerwähnt bleiben, daß sowohl die y-Methyl-, sowie die y-Äthylrhodaninsäure die kürzlich beschriebene purpurrote Farbenreaktion mit Chloralhydrat geben; die dabei entstehenden Produkte sollen noch weiter untersucht werden.

Die beschriebenen Rhodaninsäuren geben auch mit Furfurol gut krystallisierende Kondensationsprodukte, welche sich vielleicht zu einer bequemen Furfurol- respektive Pentosanbestimmung eignen, worüber Versuche im Gange sind. 